\title{
High-speed Tunable Multi-Bandpass Filter for Real-time Spectral Imaging using Blue Phase Liquid Crystal Etalon
}

\author{
Kosuke Shinatake ${ }^{\dagger}$, Takahiro Ishinabe ${ }^{\dagger}$ (member), \\ Yosei Shibata $^{\dagger}$ and Hideo Fujikake ${ }^{\dagger}$ (member)
}

\begin{abstract}
We developed a high-speed, electrically tunable multi-bandpass filter using a polymer-stabilized blue-phase liquid crystal etalon and a narrowband multi-bandpass interference filter, for use in real-time multispectral imaging systems. We show theoretically that the filter exhibits high-level transmittance $(\geq 80 \%)$ by using an Ag film as the reflective film used in the etalon. We confirmed that the filter allows for high-speed switching of the transmitted wavelength; the response time is about $1 \mathrm{~ms}$. The filter enables high-transmittance, high-speed spectroscopy. Therefore, high-sensitivity real-time multi-spectral imaging at a frame rate of $60 \mathrm{fps}$ or more can be expected in the future.
\end{abstract}

Keywords: multi-spectral imaging, liquid crystal tunable filter, blue phase liquid crystal, fabry-pérot etalon, fast switching, hightransmittance.

\section{Introduction}

Spectral imaging systems acquire object wavelength information by combining spectroscopy and imaging ${ }^{1)}$. After processing this information, the object configuration and structure can be determined by reference to its transmission, reflection, and absorption characteristics. Thus, the systems find applications in agriculture, medicine, industry, forestry, and fisheries ${ }^{2) ~ 6)}$. Recently, real-time sensing has been expected for applications such as blood glucose measurement and remote sensing with small spectral imaging systems. However, conventional spectroscopic methods such as spatial, space, and time scan systems ${ }^{7-11)}$ have a problem that it is difficult to realize all of the high-speed, small-size, and high-definition at the same time. In order to solve the problem, a method using a filter using liquid crystal (LC) has been studied. With this method, the light from an object can be obtained without degradation of spatial resolution, thus enabling a high-definition spectral imaging.

Figure 1 shows the structure of LC tunable filter (LCTF) ${ }^{7}{ }^{8)}$. This filter consists of several layers of the LC cell in which a nematic LC and a phase retardation plate

Received March 25, 2020; Revised June 3, 2020; Accepted July 14, 2020 $\dagger$ Department of Electronics Engineering, Graduate school of Engineering, Tohoku University (Sendai, Japan)

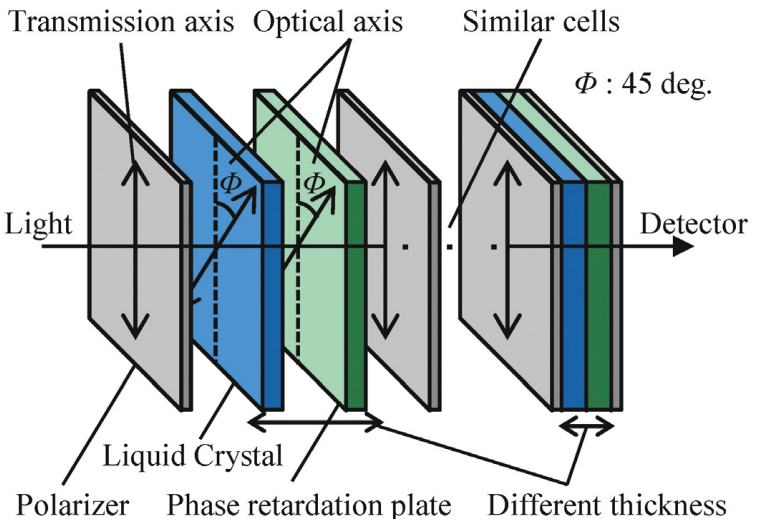

Fig. 1 Structure of conventional LCTF.

are sandwiched between polarizing plates. The optical axes of LCs and phase retardation plates are $45^{\circ}$ to the polarizer axis. Transmission wavelengths of LC cell depends on the phase retardation of the LCs and the phase retardation plates. Since the phase retardation is wavelength dependent, the LC cells has multiple transmission wavelengths. Therefore, by overlaying several LC cells and matching the transmission wavelength, only one wavelength can be transmitted. As the phase retardation of LC can be changed by electrically controlling the LC alignment, the transmission wavelength can be switched. Therefore, high-definition spectral images can be obtained by stacking the LCTF and an image sensor. However, some problems must be addressed. First, LC cells exhibit 


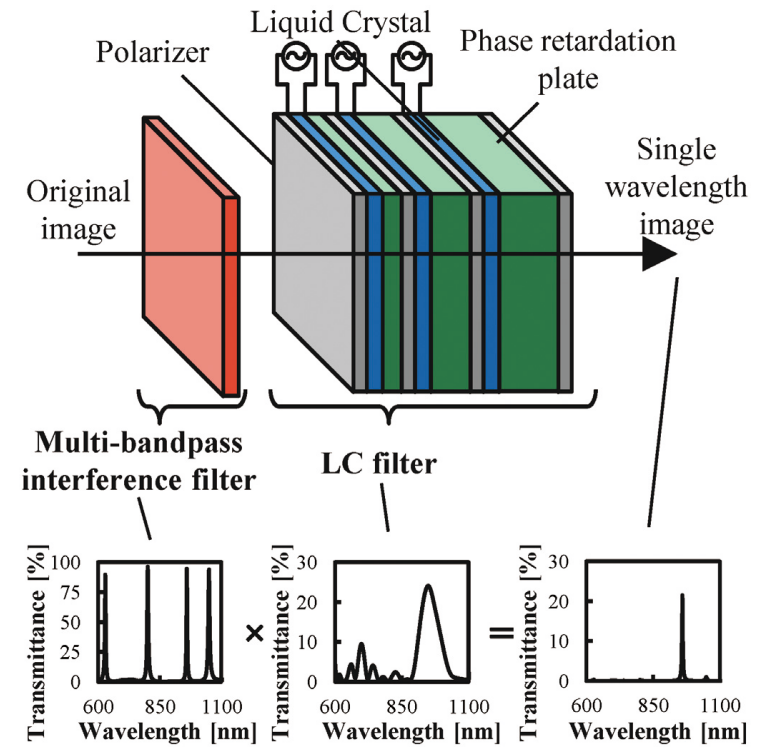

Fig. 2 Structure and principle of multi-bandpass LCTF using multi-bandpass interference filter. By multiplying the transmission spectrum of multi-bandpass interference filter and LC filter, single transmission wavelength is obtained as shown in the graphs in the lower figure.

multiple transmission wavelengths with wide full-widthat-half-maximum (FWHM) values; many cells are required to obtain a narrowband single transmission wavelength. This results in a thick filter. Second, sufficient change in phase retardation by the LC layer is required to change the transmission wavelength. Thus, the LC layer becomes thicker, and the response becomes slower due to the elasticity of the nematic LC.

To solve these problems, we previously reported a tunable multi-bandpass filter combined with a narrowband multi-bandpass interference filter and LC cells ${ }^{12)}$ 13) (Fig. 2). In this LC filter, narrowband transmission wavelength is obtained by the interference filter, and the LC layer is designed to block the wavelength other than transmission wavelength as shown in the graphs in the Fig. 2. As a result, a number of LC cell and a thickness of LC layer can be reduced. The transmission wavelength of this LC filter can be changed by applying a voltage to LC layer and switch the blocking wavelength. However, the response is not fast enough to realize high-speed spectroscopy because of the elasticity of the nematic LC. Also, the use of multiple polarizers reduces the transmittance to $25 \%$ or less, which makes imaging low-sensitivity. Thus, it is essential to switch transmission wavelengths without using polarizers. In this paper, we developed the LCTF allowing high-transmittance and high-speed switching of transmission wavelengths by using a blue-phase LC etalon.

\section{Proposal for high-speed LCTF using blue phase liquid crystal etalon}

Fabry-Pérot etalon with an LC (LC etalon) ${ }^{14)}$ is a filter that does not exploit the phase retardation of LC, it is possible to switch transmission wavelengths without using polarizer. We developed an LC etalon using a polymer-stabilized blue-phase LC 15) (PSBPLC etalon) and proposed a high-speed LCTF ${ }^{16)}$ by combining a narrowband multi-bandpass interference filter with this LC etalon for high-sensitivity real-time multi-spectral imaging. Figure 3 shows a structure and a principle of our LCTF using PSBPLC etalon. We used the narrowband multi-bandpass interference filter transmitting 960 and $1050 \mathrm{~nm}$ considering wavelengths used for near-infrared (NIR) spectroscopic measurement of glucose concentrations in aqueous solution. The FWHM of transmission spectral range was $10 \mathrm{~nm}$ and a transmittance was $90 \%$. We obtain a single transmission wavelength by matching the transmission wavelengths of two filters and the transmission wavelength of LCTF can be changed by applying a voltage to the PSBPLC etalon.

In the LC etalon, the LC layer is sandwiched between two reflective films. Incident light triggers interference between the films; only the resonant wavelength is transmitted. This wavelength is switched by modulating the refractive index of the PSBPLC. A BPLC is an LC

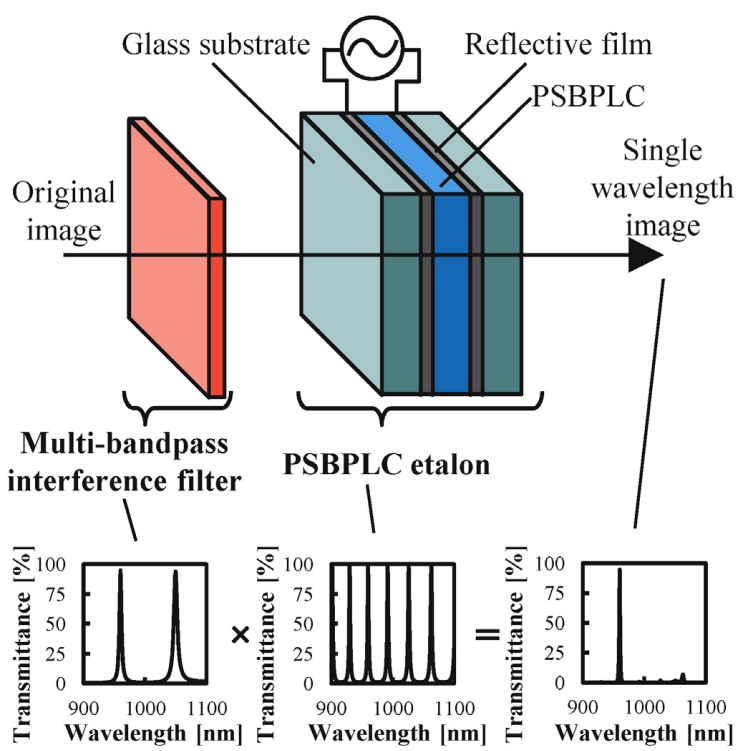

Fig. 3 Structure and principle of high-transmittance and highspeed LCTF using blue phase liquid crystal etalon. By multiplying the transmission spectrum of multi-bandpass interference filter and PSBPLC etalon, single transmission wavelength is obtained as shown in the graph in the lower figure. 
with a three-dimensional lattice structure that becomes markedly twisted on the addition of a chiral agent and the refractive index of which is identical to that of the isotropic phase when no voltage is applied. A voltage uniformly modulates the refractive index in the direction perpendicular to the electric field. The fast response speed is obtained due to the reorientation in microdomains and the strong polymer network. Thus, the PSBPLC etalon allows for high-speed and polarization-independent switching of the transmission wavelength $^{17)}$. Compact, high-speed and high-definition spectral imaging is realized by combining the PSBPLC etalon with a narrowband multi-bandpass filter.

To date, there have been reports on the advantages of the PSBPLC etalon, but no reports on the tunable multibandpass filter using the PSBPLC etalon. Here, we first investigate the characteristics that the etalon must exhibit to transmit a single wavelength with hightransmittance. Next, we explore refractive index modulation of the PSBPLC required to switch the transmission wavelength. Finally, we fabricate the LC cell and examine a response speed of PSBPLC etalon.

\section{Calculation of transmittance characteristics of the PSBPLC etalon}

The tunable multi-bandpass filter was designed to transmit the light at two wavelengths, 960 and 1,050 nm considering an application to the NIR spectroscopic measurement of glucose concentrations in aqueous solution. To ensure the transmission of only one of the two possible wavelengths of the tunable multi-bandpass filter, the PSBPLC etalon need to transmit one wavelength with high-transmittance and block the other wavelength. Figure 4 shows the required characteristics of the PSBPLC etalon. First, the peak transmittance of the PSBPLC etalon must be high at the transmission wavelength. The peak transmittance is given by the following equation:

$$
T_{\max }=\frac{(1-A)^{2}}{(1-R)^{2}}
$$

where $A$ and $R$ are the absorbance and reflectance of the reflective film, respectively. This equation shows that the peak exhibiting high-transmittance can be obtained by using a reflective film with a low-absorbance and high-reflectance.

Second, to block the other wavelength, the light should be in spectral range with low-transmittance (blocked spectral range) between peaks. For that reason,

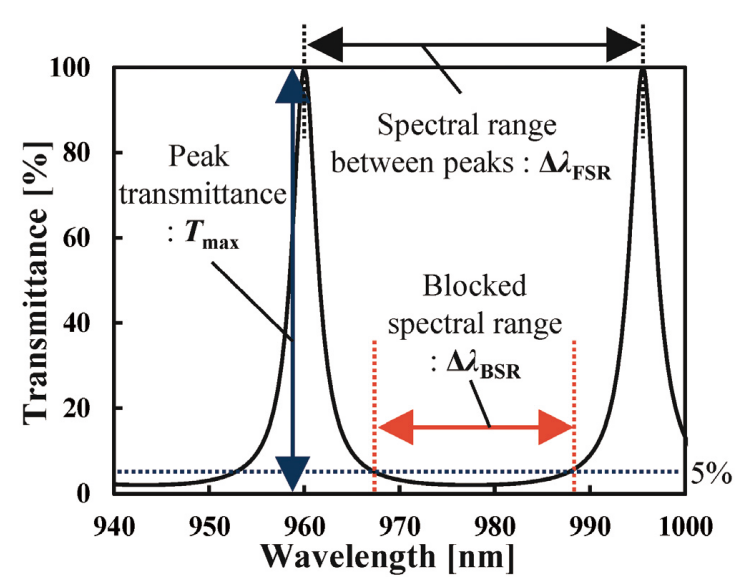

Fig. 4 Definition of required characteristics for the PSBPLC etalon to transmit only one of the two possible wavelengths of the tunable multi-bandpass filter.

blocked spectral range needs to be wide. Therefore, we investigated the conditions for a wider blocked spectral range. Since the ratio of blocked spectral range $\left(\Delta \lambda_{\mathrm{BSR}}\right)$ to the spectral range between peaks (free spectral range, $\left.\Delta \lambda_{\mathrm{FSR}}\right)$ can be treated as the probability that the wavelength to be blocked is in blocked spectral range, we evaluated $\Delta \lambda_{\mathrm{BSR}} / \Delta \lambda_{\mathrm{FSR}}$ as a parameter of the width of blocked spectral range.

Here, transmittance of the PSBPLC etalon is given by the following equation:

$$
\begin{gathered}
T=\frac{(1-A)^{2}}{(1-R)^{2}+4 R \sin ^{2}\left(\frac{\delta}{2}\right)} \\
\delta=2 \pi \frac{2 n d}{\lambda}+2 P
\end{gathered}
$$

where $\delta$ is phase difference between the light that passes through the PSBPLC etalon after one round trip and the light that passes straight through it, $n$ and $d$ are the refractive index and the thickness of PSBPLC, respectively. $\lambda$ is the wavelength and $P$ is the phase change associated with a single reflection at the reflective film. In this equation, the PSBPLC is considered to be a uniform medium with no spatial variation. According to equation (2), $\Delta \lambda_{\mathrm{BSR}} / \Delta \lambda_{\mathrm{FSR}}$ can be approximated by the ratio of the difference in $\delta$ as in equation (3), under the condition that the PSBPLC is sufficiently thick and the reciprocating optical path length of the PSBPLC etalon (2nd) is sufficiently large respect to the wavelength $(900-1,100 \mathrm{~nm})$.

$$
\frac{\Delta \lambda_{\mathrm{BSR}}}{\Delta \lambda_{\mathrm{FSR}}} \simeq \frac{\Delta \delta_{\mathrm{BSR}}}{\Delta \delta_{\mathrm{FSR}}}
$$

$\Delta \delta_{\mathrm{BSR}}$ is the difference in $\delta$ calculated from equation (2) at both ends of blocked spectral range and $\Delta \delta_{\mathrm{FSR}}$ is the 
difference in $\delta$ calculated from equation (2) at both ends of free spectral range. So, instead of $\Delta \lambda_{\mathrm{BSR}} / \Delta \lambda_{\mathrm{FSR}}$, we use $\Delta \delta_{\mathrm{BSR}} / \Delta \delta_{\mathrm{FSR}}$ to evaluate the width of blocked spectral range. By transforming $\Delta \delta_{\mathrm{BSR}} / \Delta \delta_{\mathrm{FSR}}$ using equations (1) and (2), $\Delta \lambda_{\mathrm{BSR}} / \Delta \lambda_{\mathrm{FSR}}$ is given by the following approximation.

$$
\frac{\Delta \delta_{\mathrm{BSR}}}{\Delta \delta_{\mathrm{FSR}}}=1-\frac{2}{\pi} \arcsin \sqrt{\frac{\left(1-\frac{0.05}{T_{\max }}\right)(1-R)^{2}}{4 R \frac{0.05}{T_{\max }}}}
$$

In this equation, we defined spectral ranges where the transmittance is $5 \%$ or less as blocked spectral ranges. This equation shows that a wide blocked spectral range can be obtained by using a reflective film with a highreflectance.

Here, to apply a voltage to the PSBPLC, the reflective film must have the high conductivity. On the basis of the above conditions for the reflective film, we used Ag film as the reflective film ${ }^{18}$, and optimized the absorptance and reflectance by adjusting the thickness of Ag film. We calculated the change of the peak transmittance and blocked spectral range with respect to $\mathrm{Ag}$ film thickness. We assumed that the PSBPLC and etalon glass have no wavelength dispersion of refractive index and the value is 1.5, and ignored a reflection at the boundary between the glass and the etalon exterior.

Figure 5 shows the change of the peak transmittance and blocked spectral range with respect to $\mathrm{Ag}$ film thickness. We derived the results by calculating the reflectance and absorbance from the complex refractive index and thickness of the Ag film by using equations (1) and (4). We found that the peak transmittance of more than $80 \%$ was obtained at the film thickness of $15 \mathrm{~nm}$, when the ratio of blocked spectral range was considered to be more than $50 \%$ so that more than half of spectral range would be blocked. Therefore, we found that by optimizing the Ag film thickness, only one wavelength can be transmitted with high-transmittance of $80 \%$ or more, and the other wavelength is blocked with a probability of $50 \%$ or more.

Next, to select and transmit the transmission wavelength, we need to alternately switch between the peak wavelength and blocked spectral range by using the change of transmission spectrum of the LC etalon. Therefore, we investigated the switching of the transmission wavelength by the refractive index modulation of the PSBPLC. Peak wavelengths of the etalon are given by the following equation:

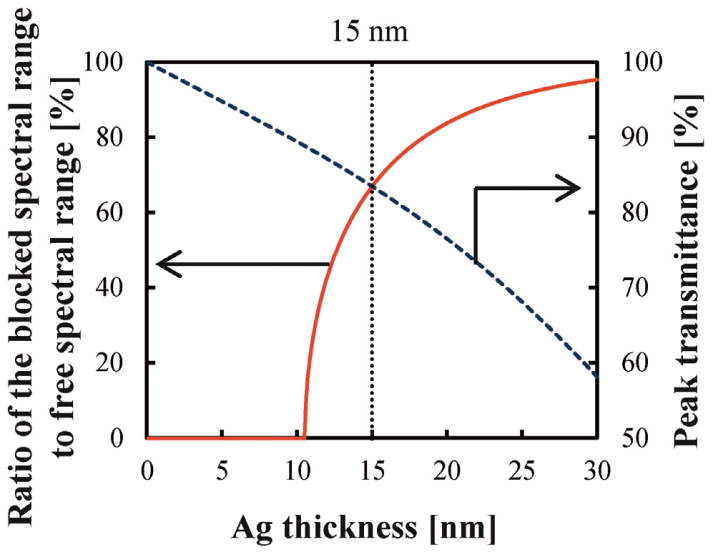

Fig. 5 Calculation result of change of blocked spectral range and peak transmittance of etalon with thickness of $\mathrm{Ag}$ film.

$$
\lambda_{\text {peak }}=\frac{2 n d}{m-P / \pi}
$$

where $m$ is an arbitrary natural number. Excluding the negligible small effect of $P$ varying with $n$, the equation shows that peak wavelengths are proportional to the refractive index of the PSBPLC. Similarly, wavelengths of blocked spectral range are proportional to the refractive index of PSBPLC. To shift peak wavelengths and wavelengths of blocked spectral range and switch the transmission wavelength, a large refractive index modulation of the PSBPLC is required. Therefore, we explored how much refractive index modulation is required to switch the transmission wavelength between 960 and 1,050 $\mathrm{nm}$. The thicker the LC layer of the etalon, the easier to switch the transmission wavelength because free spectral range becomes narrower. However, too narrow free spectral range makes blocked spectral range narrow and to block the light becomes difficult. So we investigated switching of transmission wavelength by refractive index modulation with the thickness of the LC layer being $10 \mu \mathrm{m}$. This satisfies the condition of the approximation in equation (3). We assumed that the refractive index does not exhibit wavelength dispersion in the range $900-1,100 \mathrm{~nm}$. We used a 15-nm-thick Ag film as a reflective film. The other conditions were the same as those used above.

Figure 6 shows the change in transmission wavelength in relation to the refractive index of the PSBPLC. We derived the results by using equation (2). We found that a refractive index modulation of 0.02 (from 1.478 to 1.460 ) is necessary to switch transmission wavelength from $960 \mathrm{~nm}$ to $1,050 \mathrm{~nm}$. In the next section, we examined that our PSBPLC material meets these conditions, and evaluated the switching of transmission wavelength from experimental results. 


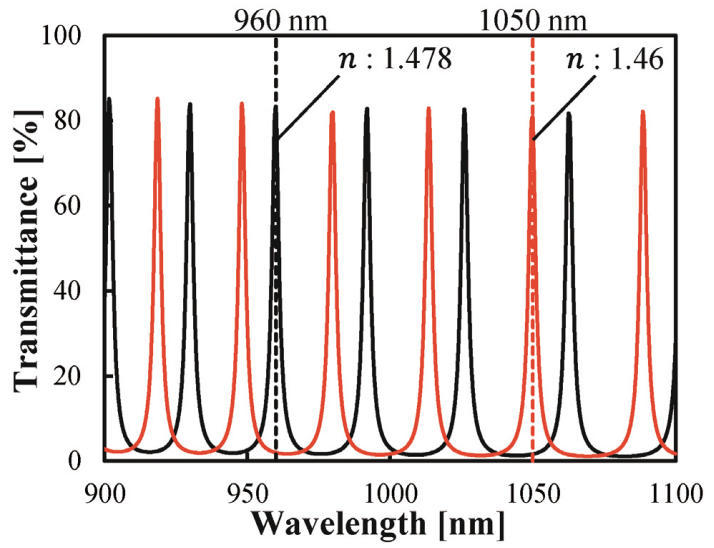

Fig. 6 Calculation result of switching transmission wavelength of the etalon by refractive index modulation of the PSBPLC.

\section{Design and evaluation of the PSBPLC etalon}

First, we examined whether our PSBPLC delivered the refractive index modulation necessary to switch the transmission wavelength. We measured peak wavelengths when a voltage was applied to the LC cell, and derived the refractive index modulation based on the change in peak wavelengths by using equation (5). We prepared Ag alloy coated glass substrates (GEOMATEC Co., Ltd.) with high oxidation resistance instead of an $\mathrm{Ag}$ film to prevents changes in optical properties due to oxidation.

To fabricate the PSBPLC, we used the LC mixture (JC-BP02, JNC Corp.), which contains chiral agents, and ultraviolet (UV)-curable monomers. We first fabricated an empty cell with a $10 \mu \mathrm{m}$ gap sustained by bead spacers and a sealing resin $(3035 \mathrm{~B}$, Three Bond Co. Ltd.). Next, JC-BP02 was injected into the cell via capillary action at a temperature of $55^{\circ} \mathrm{C}$, and the cell was then cooled at $0.1 \mathrm{~K} / \mathrm{min}$ to $44^{\circ} \mathrm{C}$. Finally, the cell was irradiated with UV light (center wavelength, 365 $\mathrm{nm})$ at $19.6 \mathrm{~mW} / \mathrm{cm} 2$ for $60 \mathrm{~s}$ to polymerize the monomers. Figure 7 shows a microscope image of the LC cell under the crossed-Nicols polarizer. We confirmed that a BPLC appeared at room temperature. We also observed platelets in the fabricated LC cell. However, there was no light scattering by the platelets, so we considered the effect on the device to be very small.

Figure 8 shows the measure results of the refractive index modulation of PSBPLC at wavelength of 1,000 nm. The broken line is the approximation derived using the extended Kerr effect ${ }^{19)}$. At an LC layer thickness of 10 $\mu \mathrm{m}$, the refractive index changed by about 0.03 (from 1.498 to 1.466 ) on application of 80 V. From these result,

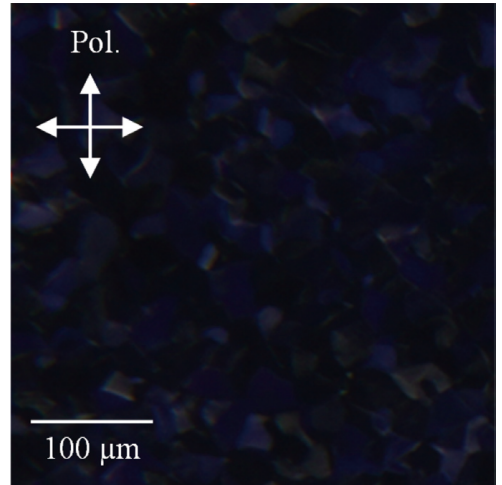

Fig. 7 Polarization micrograph of the PSBPLC at $25^{\circ} \mathrm{C}$ with no voltage applied.

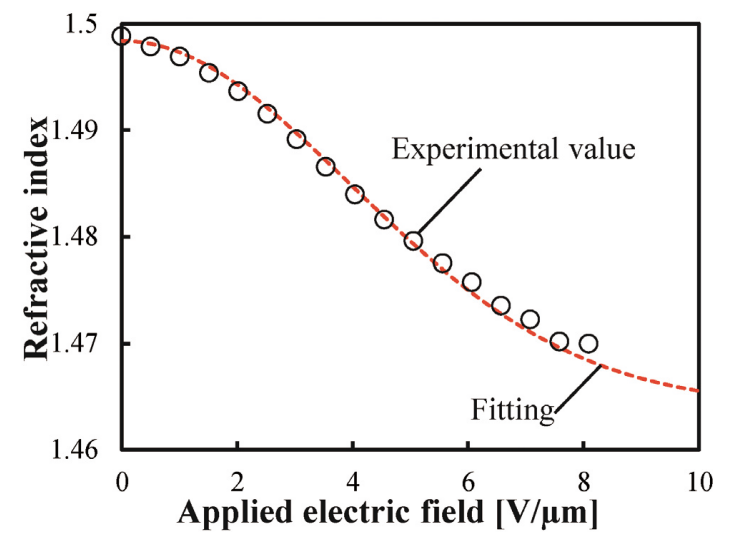

Fig. 8 Measurement result of refractive index modulation of JCBP02.

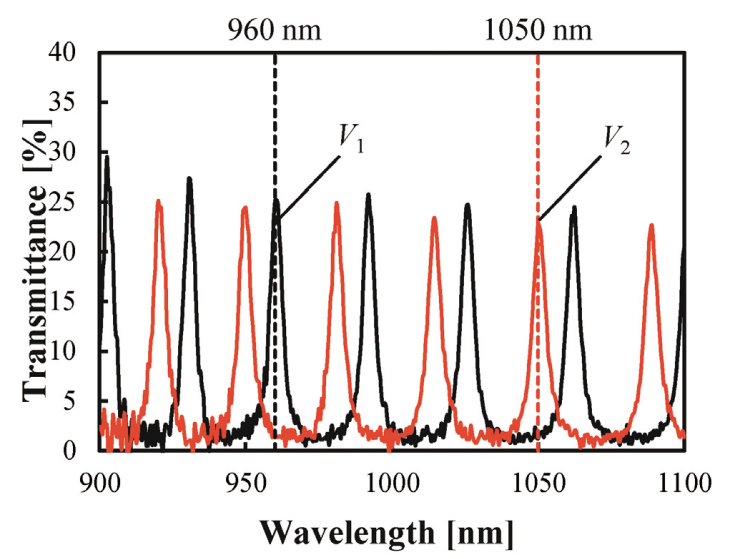

Fig. 9 Measurement result of transmission wavelength switching of PSBPLC etalon by applying voltage.

we confirmed that our PSBPLC cell meets the condition to switch transmission wavelength.

Next, we evaluated a transmission wavelength switching by using a spectrophotometer (V-770; JASCO Corp.). Figure 9 shows the change of the transmission spectrum when the voltage is switched. The peak wavelength and blocked spectral range within which the transmittance was less than $2 \%$, switched between 


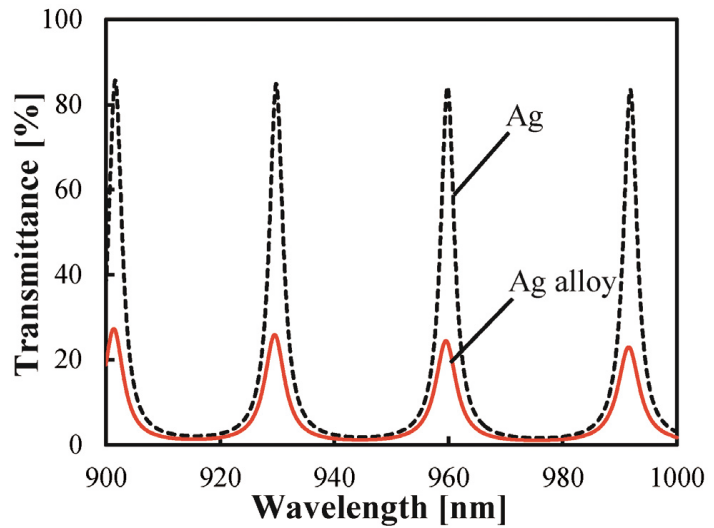

Fig. 10 Comparison of calculated transmission spectra of PSBPLC etalon used Ag or Ag alloy thin film.

wavelengths of 960 and $1,050 \mathrm{~nm}$ when the voltage changed. Therefore, we confirmed that the PSBPLC etalon sufficiently satisfies the conditions necessary for switching the transmission wavelength. However, the peak transmittance was much lower than calculation results shown in Fig. 6. To clarify the difference of transmittance between theoretical and measured values, we measured the optical properties of the $\mathrm{Ag}$ alloy thin film by using a spectroscopic ellipsometer, and found that complex refractive index differed significantly from the theoretical values.

Figure 10 compares the calculated etalon transmission spectrum between the theoretical $\mathrm{Ag}$ thin film values and the values calculated when the real optical characteristics of the Ag alloy thin film. The surface reflection of glass are included in this calculation. When the Ag alloy thin film was used, the peak transmittance fell from $84 \%$ to $24 \%$ and tended to decrease further at longer wavelengths, close to the peak transmittance in Fig. 9. The reason for this is thought to be that the absorbance of the Ag alloy thin film is greater than that of the theoretical $\mathrm{Ag}$ thin film, and the absorbance is even greater on the long-wavelength side. Although the optical characteristics of the Ag alloy are different from those of theoretical $\mathrm{Ag}$, the calculated transmission spectrum are almost in agreement with the experimental transmission spectrum in Fig. 9. Thus, the peak transmittance can be improved by using a reflective film with a complex refractive index closer to the theoretical value of $\mathrm{Ag}$.

Finally, we measured the transmission spectrum response time of the PSBPLC etalon. Figure 11 shows the transmittance and voltage waveforms used in the experiment. We used overdrive method ${ }^{20) 21)}$ to accelerate the response speed by using a waveform into which a

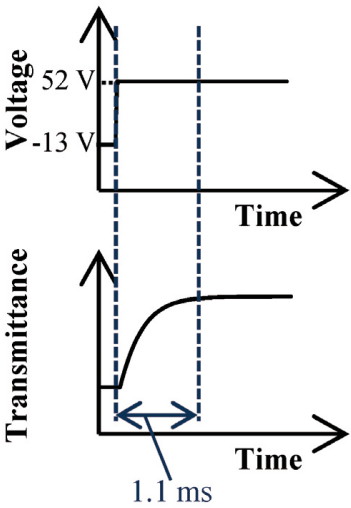

(a) Normal drive method

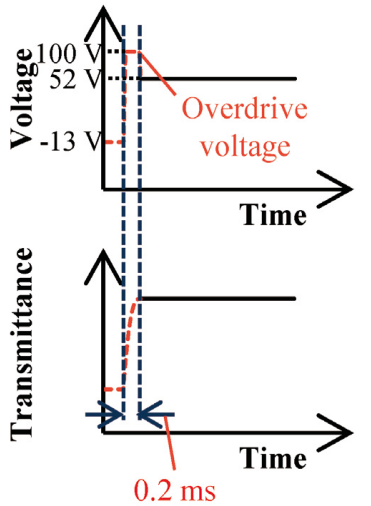

(b) Overdrive method
Fig. 11 The waveform of transmittance and voltage in overdrive method.

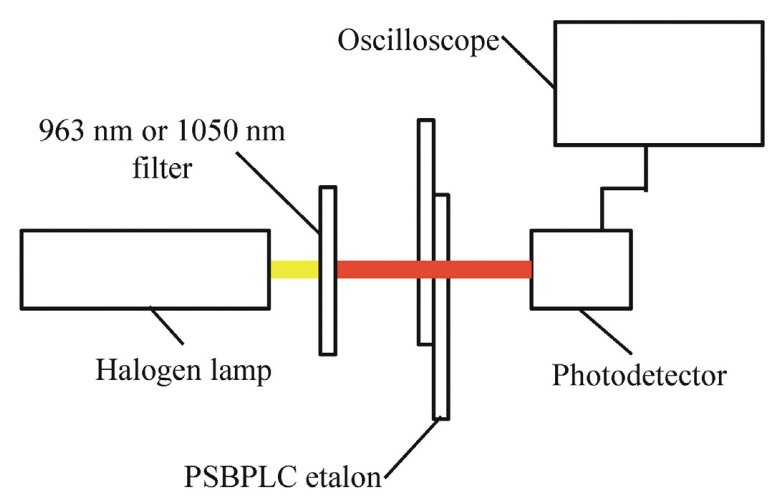

Fig. 12 Optical system for response time measurement.

higher than necessary voltage change is momentarily inserted. The voltage was varied at approximately 8.3 $\mathrm{ms}$ intervals, assuming a frame rate of $60 \mathrm{fps}$. A DC voltage of $\pm 13 \mathrm{~V}$ was applied when the transmission wavelength was $963 \mathrm{~nm}$ and $\pm 52 \mathrm{~V}$ when the transmission wavelength was $1050 \mathrm{~nm}$. In addition, as overdrive method, a voltage of $100 \mathrm{~V}$ was applied for 0.2 $\mathrm{ms}$ when the voltage was increased, and a voltage of $0 \mathrm{~V}$ was applied for $1.6 \mathrm{~ms}$ when the voltage was decreased. Figure 12 shows the optical system used for measurements. We measured changes in transmittance by using photodetector (CV-RS110; CremoVision Co., Ltd.).

Figure 13 shows the response properties at 963 and $1,050 \mathrm{~nm}$. When contemporaneous responses were compared, the fall time was shorter than the rise time. Therefore, we defined the response time as that between the moment of voltage change and the moment at which the normalized transmittance attained $90 \%$. The switching time from 963 to $1,050 \mathrm{~nm}$ was $0.2 \mathrm{~ms}$, the drive time from 1,050 to $963 \mathrm{~nm}$ was $1.1 \mathrm{~ms}$, and the response time per cycle was $1.3 \mathrm{~ms}$. Compared to the 


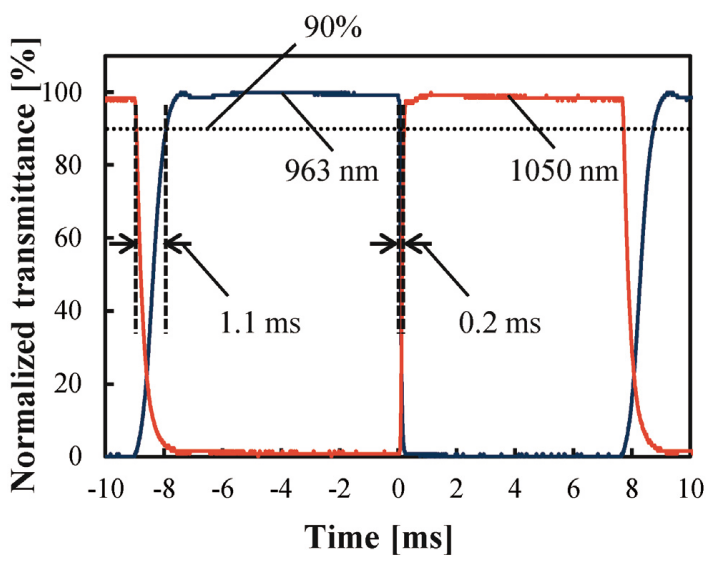

Fig. 13 Measurement results of PSBPLC etalon response time.

total response time by using the previously proposed filter shown in Fig. 2 (31.92 $\mathrm{ms})$, the response time was reduced by over $90 \%$. This will enable real-time spectral imaging at a frame rate of $60 \mathrm{fps}$ or more.

\section{Conclusion}

We proposed a high-transmittance and high-speed electrically tunable multi-bandpass filter by using a PSBPLC etalon and a multi-bandpass interference filter for application to high-sensitivity real-time spectral imaging. Calculation results showed that when an $\mathrm{Ag}$ thin film of appropriate thickness is used in the etalon as a reflective film, a high transmittance over $80 \%$ is possible, and that the transmission wavelength can be switched via a refractive index modulation of the PSBPLC. We experimentally verified the calculation results, and confirmed the transmission wavelength switching time is as low as $1.3 \mathrm{~ms}$. In the future, we will fabricate an etalon with higher-level transmittance by optimizing the reflective film.

\section{Acknowledgement}

This work was supported by JSPS KAKENHI Grant Number JP19H02187.

\section{References}

1) Y. Garini, I.T. Young and G. McNamara: "Spectral imaging: Principles and applications", Cytom. Part A, 69A, 8, pp.735-747 (Aug. 1, 2006)

2) N. Vetrekar, R. Ramachandra, K.B. Raja and R.S. Gad: "MultiSpectral Imaging for Artificial Ripened Banana Detection", 2019 8th Eur. Work. Vis. Inf. Process., pp.187-192 (2020)

3) G.N. Stamatas, M. Southall and N. Kollias: "In vivo monitoring of cutaneous edema using spectral imaging in the visible and near infrared", J. Invest. Dermatol., 126, 8, pp.1753-1760 (2006)

4) M. Khorasani, J.M. Amigo, C.C. Sun, P. Bertelsen and J. Rantanen: "Near-infrared chemical imaging (NIR-CI) as a process monitoring solution for a production line of roll compaction and tableting", Eur.J. Pharm. Biopharm., 93, pp.293-302 (2015)

5) S. Getzin, K. Wiegand and I. Schöning: "Assessing biodiversity in forests using very high-resolution images and unmanned aerial vehicles", Methods Ecol. Evol., 3, 2, pp.397-404 (Apr. 2012)

6) G. ElMasry and J.P. Wold: "High-Speed Assessment of Fat and Water Content Distribution in Fish Fillets Using Online Imaging Spectroscopy", J. Agric. Food Chem., 56, 17, pp.7672-7677 (Sep. 10, 2008)

7) K. Sato, N. Kato, S. Kano, Y. Hanazawa and T. Uchida: "Novel Multicolor Liquid Crystal Display without Color Filter", Proc.9th Int. Disp. Res. Conf., pp.392-395 (1989)

8) Y. Sato, T. Ishinabe, T. Uchida and H. Seki: "P-180L: Late-News Poster: Tunable Liquid Crystal Color Filter for Image Analysis", SID Symp. Dig. Tech. Pap., 36, 1, p. 694 (2005)

9) Y. Ohtera, T. Onuki, Y. Inoue and S. Kawakami: "Multichannel Photonic Crystal Wavelength Filter Array for Near-Infrared Wavelengths", J. Light. Technol., 25, 2, pp.499-503 (Feb. 2007)

10) M.B. Sinclair, J.A. Timlin, D.M. Haaland and M. WernerWashburne: "Design, construction, characterization and application of a hyperspectral microarray scanner", Appl. Opt., 43, 10, p. 2079 (Apr. 1, 2004)

11) Y. Garini, M. Macville, S. du Manoir, R.A. Buckwald, M. Lavi, N. Katzir, D. Wine, I. Bar-Am, E. Schröck, D. Cabib and T. Ried: "Spectral karyotyping", Bioimaging, 4, 2, pp.65-72 (June 1996)

12) Y. Fujihara, Y. Aoyagi, S. Nasuno, S. Wakashima, R. Kuroda, K. Terashima, T. Ishinabe, H. Fujikake, K. Wako and S. Sugawa: "A Multi Spectral Imaging System with a 71dB SNR 190-1100 nm CMOS Image Sensor and an Electrically Tunable Multi Bandpass Filter", ITE Trans. Media Technol. Appl., 6, 3, pp.187-194 (2018)

13) K. Terashima, K Wako, Y. Aoyagi, M.Murata, Y. Shibata, S. Sugawa, T. Ishinabe, R.Kuroda, H. Fujikake: "High speed and narrow-bandpass liquid crystal filter for real-time multi spectral imaging systems", IEICE Trans. Electron., E101C, 11, pp.897-900 (2018)

14) J.S. Patel, M.A. Saifi, D.W. Berreman, C. Lin, N. Andreadakis and S.D. Lee: "Electrically tunable optical filter for infrared wavelength using liquid crystals in a Fabry-Perot etalon", Appl. Phys. Lett., 57, 17, pp.1718-1720 (Oct. 22, 1990)

15) H. Kikuchi, M. Yokota, Y. Hisakado, H. Yang and T. Kajiyama: "Polymer-stabilized liquid crystal blue phases", Nat. Mater., 1, 1, pp.64-68 (Sep. 2, 2002)

16) K. Shinatake, T. Ishinabe, Y. Shibata and H. Fujikake: "Tunable Narrow-bandpass Filter Using Blue Phase Liquid Crystal Etalon for Real-time Multi-spectral Imaging Systems", The 26th International Display Workshops, LCTp2, pp.370-372 (Nov. 2019)

17) Y.-H. Chen, C.-T. Wang, C.-P. Yu and T.-H. Lin: "Polarization independent Fabry-Perot filter based on polymer-stabilized blue phase liquid crystals with fast response time", Opt. Express, 19, 25, p. 25441 (Dec. 5, 2011)

18) R. Ozaki, K. Nishi, T. Kan and K. Kadowaki: "Simultaneous determination of ordinary and extraordinary refractive index dispersions of nematic liquid crystals in the visible and nearinfrared regions from an interference spectrum", J. Appl. Phys., 120, 15, p. 155502 (Oct. 21, 2016)

19) J. Yan, H.C. Cheng, S. Gauza, Y. Li, M. Jiao, L. Rao and S.T. Wu: "Extended Kerr effect of polymer-stabilized blue-phase liquid crystals", Appl. Phys. Lett., 96, 7, p. 071105 (Feb. 15, 2010)

20) H. Nakamura and K. Sekiya: "51.1: Overdrive Method for Reducing Response Times of Liquid Crystal Displays", SID Symp. Dig. Tech. Pap., 32, 1, p. 1256 (2001)

21) K. Goda, I. Watanabe, E. Fukuda and K. Takatoh: "Fast response polymer stabilized blue phase by overdrive technique", Jpn.J. Appl. Phys., 59, 1, p. 014003 (2020) 


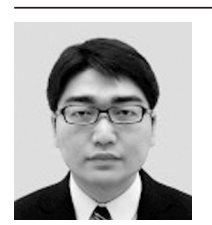

Kosuke Shinatake received the B.E. degree from Tohoku University, Sendai, Japan, in 2019. Now, he is a graduate student in the Department of Electronic Engineering, Graduate School of Engineering, Tohoku University. He is engaged in the flexible LCDs. He is engaged in researches on liquid crystal tunable filter for spectral imaging.

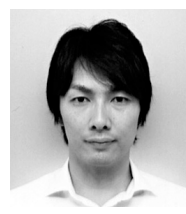

Takahiro Ishinabe received his B.S., M.S., and Ph. D. degrees in Electronic Engineering from Tohoku University, Sendai, Japan, in 1995, 1997 and 2000 , respectively. From 2000 to 2002 , he was a Research Fellow of the Japan Society for the Promotion of Science and from 2003 to 2012, he was an Assistant Professor, and since 2013, he has been an Associate Professor in the Department of Electronics, Graduate school of Engineering, Tohoku University. He has also been a Visiting Professor in the CREOL, The College of Optics and Photonics, University of Central Florida from 2010 to 2011. He has been performing a research on advanced liquid crystal displays such as wide viewing angle LCD, reflective full-color LCD, field sequential color LCD and flexible LCD.

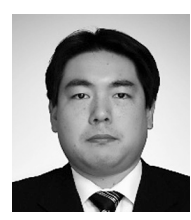

Yosei Shibata received his $\mathrm{PhD}$ degree in engineering from Tokyo Institute of Technology (Japan) in March 2013. He has research interests on organic semiconductor devices such as organic transistors and organic photovoltaics. Then he joined the National Institute of Advanced Industrial Science and Technologies (AIST, Japan) as a postdoctoral position. In October 2015, he joined the Department of Electronics of Tohoku University as an Assistant Professor.

Hideo Fujikake received M.E and Ph.D. degrees from Tohoku University, Japan, in 1985 and 2003, respectively. In 1985, he joined Japan Broadcasting Corporation (NHK). In 1988-2012, he was with NHK Science and Technology Research Laboratories. Since 2012, he has been a professor at Department of Electronic Engineering, Tohoku University. He received Best Paper Award from Institute of Electronics, Information and Communication Engineers (IEICE) in 2001, Best Paper Award from Japanese Liquid Crystal Society (JLCS) in 2001 and 2015, Niwa-Takayanagi Best Paper Awards from Institute of Image Information and Television Engineers of Japan (ITE) in 2003 and 2009, and Electronics Society Award from IEICE in 2013. His current interest is concerned with flexible liquid crystal displays. He also served as a General Vice Chair in International Display Workshops in 2015-2016, a Japan Chapter Chair in IEEE Consumer Electronics Society in 2012-2014, and a Vice President of Japanese Liquid Crystal Society in 2015-2016. He is an IEICE fellow since 2015, and ITE fellow since 2016. 